\title{
Evaluation of Posterior Ocular Structures in Patients with Isolated Iris Coloboma
}

\author{
(D) Serkan Akkaya \\ Department of Ophthalmology, Kayseri Training and Research Hospital, Kayseri, Turkey
}

\begin{abstract}
Objectives: To measure lamina cribrosa thickness (LCT), lamina cribrosa depth (LCD) and subfoveal choroidal thickness (SFCT) by imaging posterior ocular structures in pediatric cases with isolated unilateral iris coloboma and to investigate the differences as compared with healthy contralateral eyes of these cases (fellow eyes) and healthy control eyes.

Methods: This cross-sectional, comparative prospective study included seven children (age range, 9-17 years) with unilateral isolated iris coloboma. The healthy contralateral eyes of these cases formed the fellow group. An age-matched (age range 8-17 years) control group $(n=9)$, including children with both eyes having either normal or corrected-to-normal visual acuity, was formed. A detailed ophthalmic examination was performed. The posterior ocular segments were evaluated using spectral domain-optical coherence tomography (SD-OCT) with enhanced depth imaging (EDI) technique. Results: The SFCT was $372.0 \pm 48.8 \mu \mathrm{m}, 375.3 \pm 44.0 \mu \mathrm{m}$, and $386.5 \pm 71.8 \mu \mathrm{m}$, respectively, and the LCD was $362.4 \pm 68.3$ $\mu \mathrm{m}, 354.4 \pm 47.1 \mu \mathrm{m}$, and $350.7 \pm 38.1 \mu \mathrm{m}$, respectively, in the coloboma, fellow, and control eyes. There was no difference between the groups regarding SFCT and LCD. The mean LCT was significantly thinner in the coloboma eyes $(200.2 \pm 9.5$ $\mu \mathrm{m})$ than in the fellow $(238.8 \pm 26.7 \mu \mathrm{m} ; \mathrm{p}=0.023)$ and control eyes $(240.0 \pm 12.9 \mu \mathrm{m} ; \mathrm{p}<0.00 \mathrm{I})$. The LCT showed no significant correlation with age, axial length or spherical equivalent.

Conclusion: Better visual prognosis is expected in isolated iris coloboma. However, detailed examinations using new technologies, such as SD-OCT, may reveal some structural changes. Longitudinal studies are required to understand if a thinner LCT in coloboma eyes is associated with any future problems.
\end{abstract}

Keywords: Coloboma of iris, choroid, lamina cribrosa, optical coherence tomography

\section{Introduction}

Coloboma is a congenital anomaly characterized by missing pieces of tissue in the ocular structures. The estimated incidence is estimated to range from two to 14 per 100.000 births (I). During the intrauterine development process, a partial tissue defect occurs in any of the cornea, iris, ciliary body, lens, retina, choroid, and optic nerve tissues due to aberrant closure of the choroid fissure in the $5^{\text {th }} 7^{\text {th }}$ week of the fetal life (2). The anomaly presents itself within a wide spectrum from iris coloboma to clinical anophthalmia. It has heterogeneous etiology and may be genetically inher- ited; it can be seen together with chromosomal anomalies or some syndromes or may be isolated in individuals who are otherwise healthy $(I, 3)$. Ocular coloboma may be unilateral or bilateral. Visual prognosis depends on location and other accompanying conditions (2). While colobomas involving the retina or optic nerve resulting in loss of vision, iris coloboma usually does not cause loss of vision. Thus, it is considered underdiagnosed (https://ghr.nlm.nih.gov/ condition/coloboma). However, iris coloboma is the most common type of colobomas (4,5). A typical iris coloboma extends inferonasally and creates a characteristic keyhole- 
shaped pupil. Iris coloboma may either be isolated or involve other ocular tissues (6). Surgical techniques can be used for functional and cosmetic repair in iris coloboma (7).

In the literature, only a single study evaluating other ocular structures in patients with isolated iris coloboma was encountered, which was conducted by Karatepe Haşhaş et al. (8) in which the cornea was evaluated. To our knowledge, to date, there has been no study investigating the posterior segment structures in the eyes with isolated unilateral coloboma. Accordingly, the present study aimed to measure lamina cribrosa thickness (LCT), lamina cribrosa depth (LCD), and subfoveal choroidal thickness (SFCT) by imaging posterior ocular structures in pediatric cases with isolated iris coloboma and to investigate the differences as compared with healthy contralateral eyes of these cases (fellow eyes) and healthy control eyes. To our knowledge, this study is the first study to investigate posterior segment structures (LCT, LCD, and SFCT) in the eyes with isolated unilateral iris coloboma.

\section{Methods}

\section{Patients}

A detailed ophthalmic examination was performed in children who were admitted to the ophthalmology clinic due to unilateral isolated iris coloboma. Patients who had strabismus, cataract, glaucoma or other retinal disorders, patients with organic ophthalmic disorders, patients with a history of intraocular surgery, patients previously underwent laser therapy, patients who would not able to cooperate on ophthalmic examination or optical coherence tomography (OCT) were excluded from this study. Accordingly, seven children (age range, 9-17 years) with unilateral isolated iris coloboma were enrolled in this study. Healthy contralateral eyes of these children formed the fellow group. An age-matched (age range 8-17 years) control group was also formed from children $(n=9)$ who were admitted to the hospital for ophthalmic examination and in whom both eyes had normal or corrected-to-normal visual acuity (VA) (that is, the logarithm of the minimum angle of resolution [logMAR] of 0 units or better).

The present study was approved by the local ethics committee and informed consent was obtained from all participants or their parents or legal representatives. This study was conducted in accordance with the Declaration of Helsinki.

\section{Procedures}

All participants underwent detailed ophthalmic examination and the following parameters were measured: VA, spherical equivalent (SE), intraocular pressure (IOP), axial length ( $A L)$, central corneal thickness (CCT), LCT, LCD, and SFCT.
A standard Snellen chart was used to measure VA and decimal VA was converted into logMAR units. To assess the refractive status, five sequential readings were performed using a tonometer (TonoRef II; Nidek Co., Ltd; Aichi, Japan) 45 minutes after administration of an ophthalmic solution containing I\% cyclopentolate for three times with 5-minute intervals. All readings were required to be within 0.25 diopter (D) of each other. SE was calculated as the sum of spherical error and half of a cylindrical error. CCT was measured using a Scheimpflug camera (Pentacam HR; Oculus GmbH, Wetzlar, Germany). AL was measured using an IOL Master (Carl Zeiss Meditec Inc., Dublin, CA, USA). LCT and SFCT measurements were performed using spectral domain (SD)OCT device.

\section{Spectral-Domain Optical Coherence Tomography Measurements}

In all patients and controls, an SD-OCT device (Heidelberg Engineering, Heidelberg, Germany) with an enhanced depth imaging (EDI) program (HEYEX software 6.0) was used for the measurement of LCT after pupillary dilation (9) and also for the measurement of SFCT according to the procedure previously described by Spaide et al. (10). Scans with a quality score of $<20$ or poor-quality scans (those with unclear fundus images or unclear LCT borders) were excluded from the analysis both for the LCT and SFCT measurements.

To obtain the enhanced depth imaging of the optic nerve head $(\mathrm{ONH})$, the SD-OCT device was arranged in a way to form a rectangular image of $15^{\circ} \times 10^{\circ}$ centered on the optic disc. This rectangular field was then divided into nearly 65 sections, each having an average of 100 OCT frames. On these horizontal $B$ scans, three frames passing through the center, mid-upper and mid-lower aspects of the $\mathrm{ONH}$ were selected and the parameters were measured on each of these frames. The center of the lamina cribrosa plate was used for the LCT measurements. Figure I depicts the lamina cribrosa borders in an ONH OCT image.

The line combining the two ends of the Bruch's membrane was defined as the Bruch's membrane opening (BMO). All distances were measured on the line perpendicular to the reference line and the parameters were measured as closer as possible to the vertical center of the $\mathrm{ONH}$. In case a vessel precluded a measurement, the measurements were performed in the temporal region. In the horizontal SD-OCT sections, the anterior and posterior borders of the highly reflective area in the vertical center of the $\mathrm{ONH}$ were defined as the borders of lamina cribrosa and the distance between these two borders was defined as the LCT. Contrast adjustments helped with the identification of images that most clearly illustrate the lamina cribrosa. The LCD was defined as the distance between the BMO and the anterior border of the lamina cribrosa. 

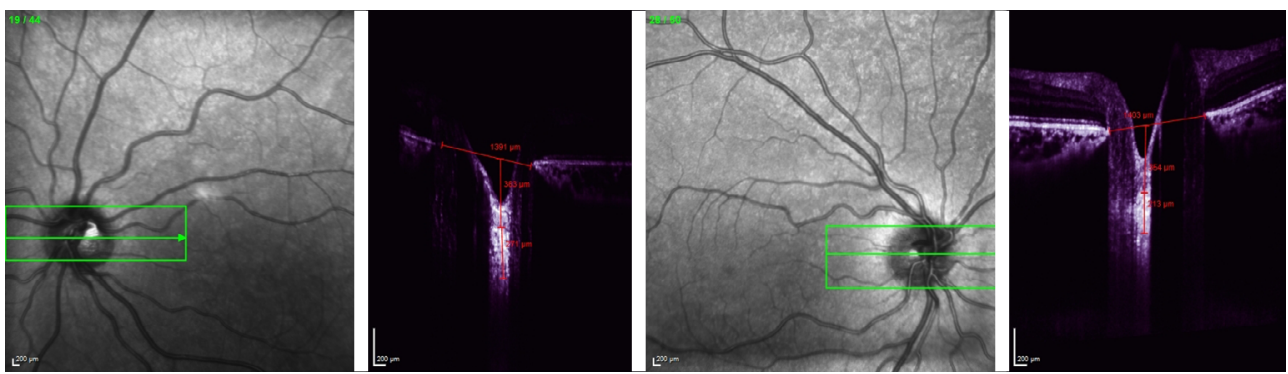

Figure I. Optical coherence tomographic images of an I I-year-old patient with unilateral isolated iris coloboma. Horizontal scans. (Left) Fellow eye of the patient with isolated iris coloboma. The thickness of the lamina cribrosa was $27 \mathrm{I} \mu \mathrm{m}$. (Right) Coloboma eye of the patient with isolated iris coloboma. The thickness of the lamina cribrosa was $213 \mu \mathrm{m}$.

The SCFT measurements were performed using the EDIOCT technique, where the SD-OCT device is pushed close to the eye to gather an inverse image. To adjust the signalto-noise ratio, seven sections, each containing 100 scans, on average were obtained within a $5^{\circ} \times 30^{\circ}$ rectangular area comprising the macula and the optic nerve. The horizontal section passing directly through the foveal center was used to measure the choroidal thickness. The choroid was measured from the outer edge of the hyperreflective line, which corresponds to the retinal pigment epithelium to the internal scleral edge. Macular choroidal thickness was measured in the subfoveal region.

\section{Statistical Analysis}

Data analyses were performed using the IBM SPSS Statistics for Windows, version 2I.0 (IBM Corp. Armonk, NY, USA). The pretest statistical power was $90 \%$. Descriptive statistics were expressed as mean \pm standard deviation (SD) for numerical variables. Multiple group comparisons of normally distributed numerical variables were performed using the Analysis of Variance (ANOVA) test. The Tukey test was used for post-hoc comparisons.

Pearson's correlation coefficient was used to determine the relationship between numerical variables. The level of statistical significance was set at a $p$-value of $<0.05$.

\section{Results}

In this study, seven eyes with isolated iris coloboma and their fellow eyes and 9 control eyes were included in the analysis. The mean age of the patient group ( 5 females and 2 males) was $13.14 \pm 3.93$ years and the mean age of the control group (7 females and 2 males) was $13.11 \pm 3.95$ years. The patient and control groups did not differ concerning distributions of age and $\operatorname{sex}(p=0.98$ and $p=0.77)$. The coloboma, fellow, and control eye groups did not differ concerning their ocular characteristics (IOP, VA, SE, AL, and CCT) (Table I).

Evaluation of the posterior ocular regions of the eyes revealed that the coloboma eyes did not differ from the fellow and control eyes in terms of the mean SFCT and LCD. On the other hand, the mean LCT was significantly thinner in the coloboma eyes $(200.2 \pm 9.5 \mu \mathrm{m})$ as compared with the fellow $(238.8 \pm 26.7 \mu \mathrm{m})$ and control $(240.0 \pm 12.9 \mu \mathrm{m})$ eyes $(p=0.023$ and $p<0.00 I$, respectively; Table 2$)$.

The correlation analysis performed to evaluate the relationship of LCT with age, AL, and SE revealed that the LCT was not significantly correlated with age, $A L$ or $S E$ in the coloboma, fellow, and control eyes (Table 3).

Table I. Results of comparison of ocular characteristics of coloboma, fellow and control eyes

\begin{tabular}{lcccrr} 
& $\begin{array}{c}\text { Coloboma eyes } \\
(\mathbf{n}=\mathbf{7})\end{array}$ & $\begin{array}{c}\text { Fellow eyes } \\
(\mathbf{n}=\mathbf{7})\end{array}$ & $\begin{array}{c}\text { Control eyes } \\
(\mathbf{n}=\mathbf{9})\end{array}$ & p2 & p3 \\
\hline IOP, mmHg & $13.0 \pm 1.0$ & $13.5 \pm 2.2$ & $13.8 \pm 2.0$ & 0.83 & 0.62 \\
VA, logMAR & $0.02 \pm 0.04$ & $-0.01 \pm 0.03$ & $-0.02 \pm 0.04$ & 0.18 & 0.94 \\
SE, D & $0.81 \pm 0.64$ & $0.66 \pm 0.28$ & $0.24 \pm 0.46$ & 0.83 & 0.93 \\
AL, mm & $22.29 \pm 0.52$ & $22.57 \pm 0.76$ & $22.64 \pm 0.53$ & 0.69 & 0.07 \\
CCT, $\mu \mathrm{m}$ & $559.00 \pm 27.86$ & $543.80 \pm 25.87$ & $544.44 \pm 27.32$ & 0.65 & 0.97 \\
\hline
\end{tabular}

IOP, intraocular pressure;VA: visual acuity; logMAR: the logarithm of the minimum angle of resolution; D: diopter; SE: spherical equivalent; AL: axial length; CCT: central corneal thickness. pl: Coloboma vs. fellow eyes, p2: Coloboma vs. control eyes, p3: Fellow vs. control eyes. 
Table 2. Results of measurements of subfoveal choroidal thickness, lamina cribrosa thickness, and lamina cribrosa depth in coloboma, fellow and control eyes

\begin{tabular}{lccccr} 
Coloboma eyes & Fellow eyes \\
$(\mathbf{n = 7})$ & $(\mathbf{n = 7})$ & $\begin{array}{c}\text { Control eyes } \\
(\mathbf{n = 9})\end{array}$ & p1 & p3 \\
\hline SFCT, $\mu \mathrm{m}$ & $372.0 \pm 48.8$ & $375.3 \pm 44.0$ & $386.5 \pm 71.8$ & 0.99 & 0.88 \\
$\mathrm{LCT}, \mu \mathrm{m}$ & $200.2 \pm 9.5$ & $238.8 \pm 26.7$ & $240.0 \pm 12.9$ & 0.023 & 0.93 \\
$\mathrm{LCD}, \mu \mathrm{m}$ & $362.4 \pm 68.3$ & $354.4 \pm 47.1$ & $350.7 \pm 38.1$ & 0.95 & 0.99 \\
\hline
\end{tabular}

SFCT: subfoveal choroidal thickness; LCT: lamina cribrosa thickness; LCD: lamina cribrosa depth; pI: Coloboma eyes vs. fellow eyes, p2: Coloboma vs. control eyes, p3: Fellow vs. control eyes.

Table 3. Correlation of LC thickness values with the age, axial length, and SE in coloboma, fellow and control eyes

\section{LCT}

\begin{tabular}{|c|c|c|c|c|c|c|}
\hline & \multicolumn{2}{|c|}{ Coloboma eyes } & \multicolumn{2}{|c|}{ Fellow eyes } & \multicolumn{2}{|c|}{ Control eyes } \\
\hline & $\mathbf{r}$ & $\mathbf{p}$ & $\mathbf{r}$ & $\mathbf{p}$ & $\mathbf{r}$ & $\mathbf{p}$ \\
\hline Age & -0.60 & 0.15 & -0.14 & 0.76 & -0.53 & 0.14 \\
\hline$A L$ & 0.58 & 0.22 & -0.71 & 0.11 & 0.12 & 0.74 \\
\hline SE & -0.15 & 0.74 & 0.66 & 0.10 & -0.35 & 0.34 \\
\hline
\end{tabular}

LCT: Lamina cribrosa thickness;AL: axial length; SE: spherical equivalent.

\section{Discussion}

In children with coloboma, the prognosis is affected by the eye sections involved. Poorer prognosis has been reported for colobomas accompanied by microcornea, microphthalmia, cysts, and other abnormalities, whereas simple coloboma has been reported to have the best prognosis (I I). Studies evaluating other structures of the eye in patients with isolated iris coloboma are quite limited in number. In their study, Karatepe Haşhaş et al. (8) evaluated the cornea in patients $(n=9)$ with isolated iris coloboma and reported differences in several parameters, including lower keratometry values, increased corneal thickness, reduced anterior chamber depth, greater pupil diameter, and smaller horizontal corneal diameter in the eyes with coloboma as compared with completely normal contralateral (fellow) eyes. In the present study, although the CCT tended to be higher in the eyes with unilateral iris coloboma as compared with their contralateral (fellow) eyes, this difference failed to reach the level of statistical significance.

In the present study, the mean LCT among the posterior segment parameters was significantly thinner in the eyes with isolated iris coloboma as compared with the fellow and healthy control eyes. Although the mean LCD was higher in the eyes with coloboma as compared with the fellow and control eyes, the difference did not reach the level of statistical significance. The mean SFCT was also lower in the coloboma eyes as compared with the fellow and control eyes; however, the difference was not statistically significant.

Lamina cribrosa is the region at which the optic nerve fibers exit the eye through a hole in the posterior sclera and through which central retinal vessels pass (II). Until recently, data on the structural and histological features of lamina cribrosa have been based on the information obtained from animal studies, cadavers, and enucleated eyes and there was no opportunity to conduct a prospective examination in particular. The opportunity for in vivo investigation of ocular structures has been provided along with the introduction of OCT $(I I, I 2)$. The sensitivity of imaging the deep ocular layers has been enhanced with the use of the EDI feature of the SDOCT. Thus, better visualization of the posterior segments and lamina cribrosa has become possible $(1 \mathrm{I}, 13,14)$. In the EDIOCT technique, the OCT device is pushed towards the eye close enough and detailed cross-sectional images of the lamina cribrosa, including the anterior laminar surface, the laminar pores, and the neuroretinal rim, are obtained (II).

Structural and histological changes in the lamina cribrosa are investigated in ocular diseases and in some systemic diseases with ocular involvement (I5-22). Particularly, based on the consideration that it plays a role in the pathogenesis 
in patients with glaucoma, changes in the lamina cribrosa are investigated $(15,16)$. It has been suggested that quantitative changes determined by OCT can be used as biomarkers in predicting glaucoma-related injury (I7). Early detection of changes in the lamina cribrosa would enable the early diagnosis of glaucoma (18). Increased severity of glaucoma was associated with increased LCD and decreased LCT (19). LCT was low and LCD was high in migraine patients either with or without aura and these findings suggested to indicate that migraine patients are at risk of developing glaucoma (20). LCT was lower in patients with retinal vein occlusion as compared with healthy individuals (2I). In a study evaluating the lamina cribrosa using OCT, a thicker and more anteriorly positioned lamina cribrosa was demonstrated in patients with diabetes mellitus as compared with healthy controls (22).

A study conducted in healthy adults ( $\geq 18$ years old) reported a positive correlation between LCT and age (23). In the present study, no correlation was determined between age and LCT either in the coloboma cases or in the controls. This might have resulted from the study participants' consisting of pediatric cases within a limited age range. I think that repeating the measurements in adults and within a wider age range would be beneficial. Moreover, in the present study, the LCT was not found to be correlated with AL or SE. In their study conducted in healthy adults, Xio et al. (23) found no correlation between LCT and AL. Likewise, Lee et al. (24) found that the LCT was not correlated with CCT or AL.

In brief, the present study found that the mean LCT was significantly lower in coloboma eyes of patients with isolated iris coloboma as compared with their fellow eyes and healthy control eyes. The above-mentioned studies have suggested that there is a relationship between LCT and ophthalmic or certain systemic diseases. Based on this suggestion, I could speculatively propose that some other pathologies associated with low LCT may occur in this cases in the future. Longitudinal studies, including larger populations, are required to confirm this relationship.

Limited patient number can be considered a limitation of the present study; however, isolated unilateral iris coloboma is already a rare condition. Nevertheless, the pretest statistical power of the present study is $90 \%$ and this is adequate for the present study to be worthy of note.

\section{Conclusion}

A better visual prognosis is expected for patients with isolated iris coloboma. However, detailed examinations performed using new technologies, such as SD-OCT may reveal some structural changes, that have not yet caused clinical problems. In the present study, the LCT was lower in the coloboma eyes as compared with the fellow and control eyes. Longitudinal studies, including larger populations, are required to understand whether this is associated with some other future problems.

\section{Disclosures}

Ethics Committee Approval: The present study was approved by the local ethics committee and informed consent was obtained from all participants or their parents or legal representatives. This study was conducted in accordance with the Declaration of Helsinki.

Peer-review: Externally peer-reviewed.

Conflict of Interest: None declared.

\section{References}

I. Ohuchi H, Sato K, Habuta M, Fujita H, Bando T. Congenital eye anomalies: more mosaic than thought? Congenit Anom (Kyoto) 2019;59:56-73. [CrossRef]

2. Onwochei BC, Simon JW, Bateman JB, Couture KC, Mir E. Ocular colobomata. Surv Ophthalmol 2000;45:175-94. [CrossRef]

3. Vogt G, Puhó E, Czeizel AE. A population-based case-control study of isolated ocular coloboma. Ophthalmic Epidemiol 2005; 12:19|-7. [CrossRef]

4. Uhumwangho OM, Jalali S. Chorioretinal coloboma in a paediatric population. Eye (Lond) 20I4;28:728-33. [CrossRef]

5. Nakamura KM, Diehl NN, Mohney BG. Incidence, ocular findings, and systemic associations of ocular coloboma: a population-based study. Arch Ophthalmol 201 I;129:69-74. [CrossRef]

6. Gregory-Evans CY, Williams MJ, Halford S, Gregory-Evans K. Ocular coloboma: a reassessment in the age of molecular neuroscience. J Med Genet 2004;41:88I-91. [CrossRef]

7. Cionni RJ, Karatza EC, Osher RH, Shah M. Surgical technique for congenital iris coloboma repair. J Cataract Refract Surg 2006;32:19/3-6. [CrossRef]

8. Karatepe Haşhaş AS, Arifoğlu HB, Yüce Y, Duru N, Ulusoy DM, Zararsiz G. Evaluations of corneas in eyes with isolated iris coloboma. Curr Eye Res 2017;42:4I-6. [CrossRef]

9. Hornby SJ, Adolph S, Gilbert CE, Dandona L, Foster A. Visual acuity in children with coloboma: clinical features and a new phenotypic classification system. Ophthalmology 2000; 107:5 I I-20.

10. Spaide RF, Koizumi H, Pozzoni MC. Enhanced depth imaging spectral-domain optical coherence tomography. Am J Ophthalmol 2008; | 46:496-500. [CrossRef]

II. Park SC, Ritch R. High resolution in vivo imaging of the lamina cribrosa. Saudi J Ophthalmol 201 I;25:363-72. [CrossRef]

12. Adhi M, Duker JS. Optical coherence tomography-current and future applications. Curr Opin Ophthalmol 2013;24:213-2I.

13. Lee EJ, Kim TW, Weinreb RN, Park KH, Kim SH, Kim DM. Visualization of the lamina cribrosa using enhanced depth imaging spectral-domain optical coherence tomography. Am J Ophthalmol 20I I;152:87-95.el. [CrossRef]

14. Nuyen B, Mansouri K, Weinreb RN. Imaging of the lamina cribrosa using swept-source optical coherence tomography. J Curr Glaucoma Pract 2012;6:1 I3-9. [CrossRef] 
15. Park SC. In vivo evaluation of lamina cribrosa deformation in glaucoma. J Glaucoma 2013;22 (Suppl 5):S29-3I. [CrossRef]

16. Quigley HA. The contribution of the sclera and lamina cribrosa to the pathogenesis of glaucoma: Diagnostic and treatment implications. Prog Brain Res 2015;220:59-86. [CrossRef]

17. Abe RY, Gracitelli CPB, Diniz-Filho A, Tatham AJ, Medeiros FA. Lamina Cribrosa in glaucoma: diagnosis and monitoring. Curr Ophthalmol Rep 2015;3:74-84. [CrossRef]

18. Sigal IA, Wang B, Strouthidis NG, Akagi T, Girard MJA. Recent advances in OCT imaging of the lamina cribrosa. Br J Ophthalmol. 2014;98 (Suppl 2):ii34-9. [CrossRef]

19. Kim M, Bojikian KD, Slabaugh MA, Ding L, Chen PP. Lamina depth and thickness correlate with glaucoma severity. Indian J Ophthalmol 2016;64:358-63.

20. Sirakaya E, Kucuk B, Agadayi A, Yilmaz N. Evaluation of the lamina cribrosa thickness and depth in patients with migraine.
Int Ophthalmol 2020;40:89-98. [CrossRef]

2I. Lim SH, Kim M, Chang W, Sagong M. Comparison of the lamina cribrosa thickness of patients with unilateral branch retinal vein occlusion and healthy subjects. Retina 2017;37:515-21. [CrossRef]

22. Akkaya S, Küçük B, Doğan HK, Can E. Evaluation of the lamina cribrosa in patients with diabetes mellitus using enhanced depth imaging spectral-domain optical coherence tomography. Diab Vasc Dis Res 2018;15:442-8. [CrossRef]

23. Xiao $H, X u X Y$, Zhong YM, Liu X. Age related changes of the central lamina cribrosa thickness, depth and prelaminar tissue in healthy Chinese subjects. Int J Ophthalmol 2018; I I:1842-7.

24. Lee EJ, Kim TW, Weinreb RN, Suh MH, Kim H. Lamina cribrosa thickness is not correlated with central corneal thickness or axial length in healthy eyes: central corneal thickness, axial length, and lamina cribrosa thickness. Graefes Arch Clin Exp Ophthalmol 2013;251:847-54. [CrossRef] 